no se visualizan células neoplásicas. De ahí que, ante una lesión de aspecto tumoral con citología urinaria negativa, se debe pensar en la posibilidad de que nos encontremos ante un caso de amiloidosis primitiva ${ }^{6}$. La visión endoscópica de placas amarillentas también puede hacernos sospechar la presencia de una amiloidosis. Esta enfermedad vesical primaria puede evolucionar de una forma lenta afectándose progresivamente toda la pared de la vejiga incluyendo los nervios, originando una neuropatía sensitiva que conlleva a un trastorno de vaciado de la vejiga. Así, en etapas tardías de la enfermedad se comporta como una vejiga neurógena. El examen anatomo patológico revelará la ausencia de neoplasia. Los depósitos de amiloide generalmente invaden los vasos y el tejido conectivo submucoso, estando menos frecuentemente afectada la muscular ${ }^{7}$. La sustancia amiloidea teñida con Rojo Congo ${ }^{3}$ adopta birrefringencia verdosa observada con luz polarizada. Si se tiñe con tioflavina $\mathrm{T}$ también presenta birrefringencia. Resulta interesante la tipificación inmunohistoquímica con anticuerpos específicos contra los diferentes componentes del amiloide para el diagnóstico ${ }^{8}$. La mayoría de las formas localizadas contienen, además de la proteína amiloide $\mathrm{A}$, depósitos de cadenas ligeras de inmunoglobulinas monoclonales (de tipo AL). Los depósitos exclusivamente formados por proteína A (de tipo AA) sugieren amiloidosis secundaria.

Otras pruebas útiles para diferenciar la enfermedad primaria de la sistémica son la biopsia de la mucosa rectal y grasa abdominal, pruebas funcionales orgánicas como creatinina, proteinuria, transaminasas, etc. y el ecocardiograma.

En cuanto al tratamiento, se recomienda en estos casos de amiloidosis vesical localizada una resección transuretral amplia, la cual facilita tras la intervención endoscópica un vaciamiento lento de la sustancia amiloide intersticial, lo que ayudará a la curación. Una vez realizada la resección endoscópica, puede resultar útil el tratamiento local con dimetilsulfóxido (DMSO) en instilaciones vesicales de $50 \mathrm{ml}$ al $50 \%$ durante $30 \mathrm{~min}$, repitiéndose cada 15 días $^{9}$.

La colchicina también se emplea como tratamiento oral a largo plazo en caso de amiloidosis a dosis de 0,5 mg una o 2 veces al día ${ }^{1}$. Está descrita la ligadura de las arterias hipogástricas, incluso la cistectomía para casos extremos en los que se ha de recurrir a tratamientos más agresivos ${ }^{3}$.

A su vez, se exige un estudio y un seguimiento prolongado de por vida mediante cistoscopias en estos pacientes debido a la frecuencia de multiples recurrencias ${ }^{9}$ y en ocasiones, en busca de una amiloidosis sistémica que pudiera presentarse de forma secundaria ${ }^{10}$.

B I B L I O G R A F Í A

1. Jiménez Cruz JF, Rioja Sanz LA. En: Tomo I, editor. Otras cistopatías. Tratado de Urología. Barcelona: J.R. Prous Editores; 1993:655.

2. González Naranjo F, Velasco Alonso J, Méndez López M, Escaf Barmadah S, González Tuero JS, Capdevila Hernández J. Bladder amyloidosis. Actas Urol Esp. 1992;16:579-81.

3. Caballero Giné JM, Borrat Font P, Martí Picas L, Ristol Pont J. Amiloidosis vesical: Presentación de dos casos. Actas Urol Esp. 2000;24:413-5.

4. Nurmi MJ, Ekfors TO, Puntala PV. Secondary amyloidosis of the bladder: a cause of massive hematuria. J Urol. 1987;138: 44-5.

5. Tesedo Cubero J, Ristol RM, Fernández Zuazu J, Dinares J, Autanell J. Massive hematuria and bladder perforation, complication of systemic amyloidosis. Arch Esp Urol. 1991;44:862-4.

6. Begara Morillas F, Silmí Moyano A, Espinosa Fernández B, Prieto Chiparro L, Rapariz González M, González Morales ML, et al. Bladder amyloidosis in a female patient with suspected bladder carcinoma. Arch Esp Urol. 1994;47:176-9.

7. Caldamone AA, Elbadawi A, Moshtagi A, Frank IN. Primary localizad amyloidosis of urinary bladder. Urology. 1980;15: 174-180.

8. Linke RP. Highly sensitive diagnosis of amyloid and various amyloid syndromes using Congo red fluorescence. Virchows Arch. 2000;436:439-48.

9. Nurmi MJ, Ekfors TO, Rajala PO, Puntala PV. Intravesical dimethyl sulfoxide instillations in the teratment of secondary amyloidosis of the bladder. J Urol. 1990;143:808-10.

10. Malek RS, Wahner-Roedler DL, Gertz MA, Kyle RA. Primary localized amyloidosis of the bladder: experience with dimethyl sulfoxide therapy. J Urol. 2002;168:1018-20.

N. Senarriaga Ruiz de la Illa*, A. Loizaga Iriarte, I. Lacasa Viscasillas y M. Unda Urzaiz

Servicio de Urología, Hospital de Basurto, Universidad del País Vasco, Bilbao, España

*Autor para correspondencia.

Correo electrónico: nerea.senarriagaruiz@osakidetza.net (N. Senarriaga Ruiz de la Illa).

\title{
Construcción del ombligo en la extrofia vesical
}

\section{Umbilical reconstruction in patients with vesical extrofia}

\section{Sr. Director:}

La ausencia de ombligo tras la cirugía reconstructiva de la extrofia vesical constituye un defecto que agrava, el ya normalmente pobre aspecto estético de la pared abdominal de estos pacientes. La técnica de la reconstrucción umbilical «tipo canguro» ${ }^{1}$, realizada mediante un colgajo cutáneo de base inferior, constituye una técnica sencilla y eficaz, que 


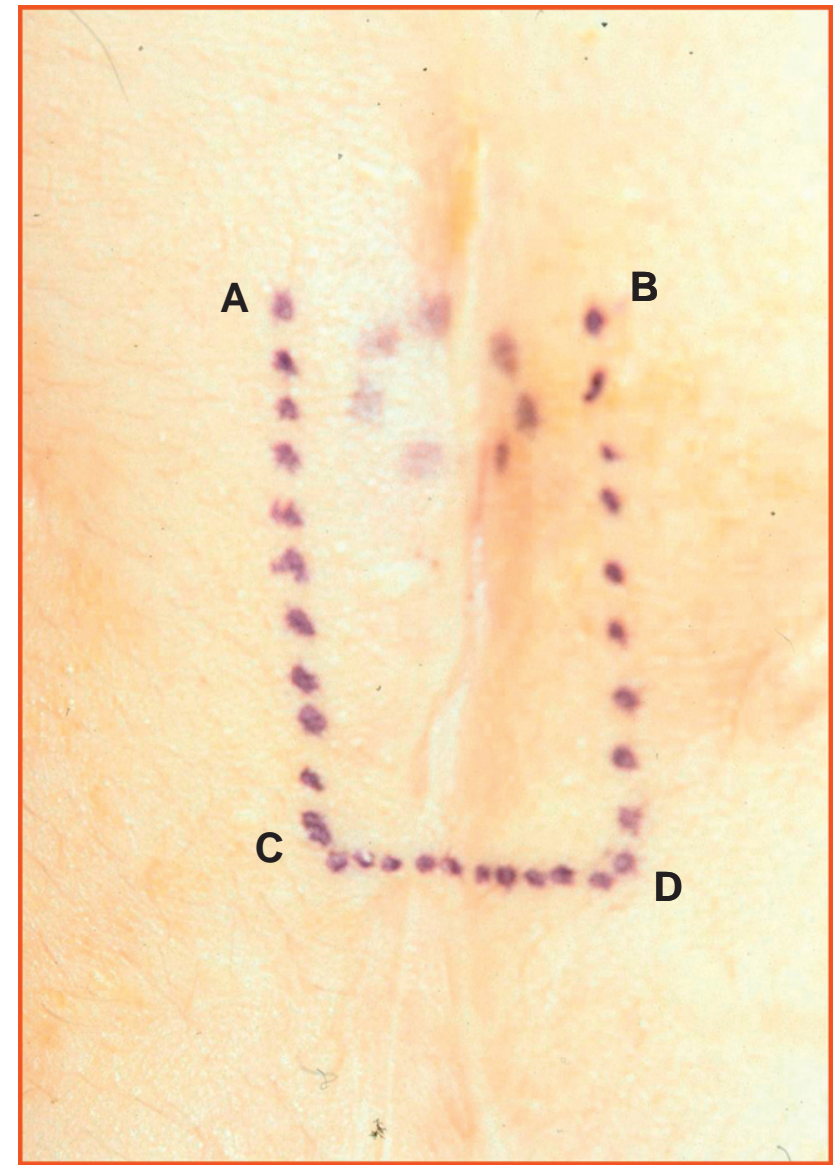

Figura 1 - Dibujo del colgajo cutáneo de base superior y de $2 \times 3 \mathrm{~cm}$ que constituirá el futuro ombligo.

permite dotar de ombligo a los pacientes con ausencia de éste.

Presentamos el caso de 4 pacientes afectos de extrofia vesical, 1 niño y 3 niñas, de edades comprendidas entre 6-16 años de edad se les construyó un nuevo ombligo. Para la reconstrucción, se marcó el punto del futuro ombligo en el centro de una línea que unía ambas crestas ilíacas. La localización final de éste se decidió tras comprobar en decúbito y en posición ortostática cual era el punto más adecuado. Posteriormente se dibujó un colgajo cutáneo pediculado, de $2 \times 3 \mathrm{~cm}$ que tomó como base el punto marcado previamente (figs. 1 y 2). Tras doblar por la mitad dicho colgajo y suturar ambos bordes laterales se obtuvo una pequeña bolsa que se fijó al plano subyacente. La intervención se completó al cerrar los bordes de la herida sobre la bolsa. El resultado estético ha sido favorable en todos los pacientes, aunque el primero de la serie, en el que las medidas del colgajo fueron algo más económicas, presentó un cierto grado de retracción.

Ha habido otras técnicas ${ }^{2-4}$ descritas hasta ahora para la reconstrucción del ombligo, pero ninguna tan sencilla y con tan poca cicatriz adicional. El esfuerzo operatorio que representa la construcción de un nuevo ombligo, ya sea en un acto quirúrgico aislado, como en nuestros casos, o en el curso de otro acto quirúrgico ${ }^{2,4}$, está plenamente justificado por la importancia social que el ombligo tiene para el paciente y su familia.

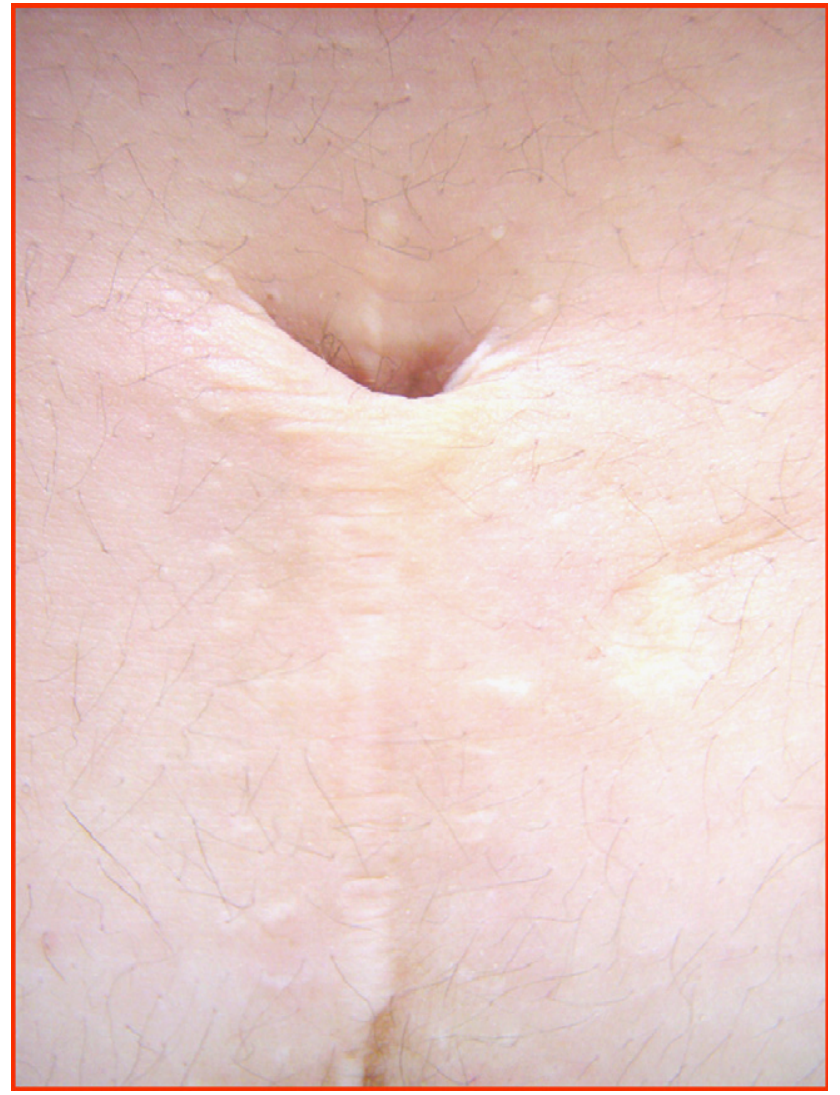

Figura 2 - Aspecto final varios meses después de la reconstrucción.

En nuestra casuística no se ha tenido ocasión de ensayar la colocación del cabo cutáneo de una apendicovesicostomía tipo Mitrofanoff en el fondo de la bolsa, como se ha hecho en otras técnicas descritas ${ }^{2,4}$, pero sería factible y evitaría los problemas derivados de la exposición al aire de la mucosa apendicular.

B I B L I O G R A F Í A

1. Feyaerts A, Mure PY, Jules JA, Morel-Journel N, Mouriquand P. Umbilical reconstruction in patients with exstrophy: The kangaroo pouch technique. J Urol. 2002;165:2026-8.

2. Sumfest JM, Mitchel ME. Reconstruction of the umbilicus in exstrophy. J Urol. 1994;151:453-4.

3. Hanna MK, Ansong K. Reconstruction of umbilicus in bladder exstrophy. Urology. 1984;24:324-5.

4. Pinto PA, Stock JA, Hanna MA. Results of umbilicoplasty for bladder exstrophy. J Urol. 2000;164:2055-7.

\section{J. Rodó Salas ${ }^{\mathrm{a}, *}$ y M. Olivares Muñoz ${ }^{\mathrm{b}}$}

${ }^{a}$ Sección de Urología Pediátrica, Hospital Sant Joan de Déu, Barcelona, España

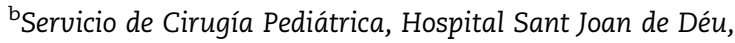
Barcelona, España

*Autor para correspondencia.

Correo electrónico: jrodo@hsjdbcn.org (J. Rodó Salas). 